\title{
High Resolution Atomic Force Microscopy Visualization of Fibrinogen Unfolding on Modified Graphite
}

\author{
E. V. Dubrovin ${ }^{1,2}$, N. A. Barinov ${ }^{1}$ and D. V. Klinov ${ }^{1}$ \\ 1. Federal Research and Clinical Center of Physical-Chemical Medicine, Malaya Pirogovskaya, \\ 1a, Moscow 119435, Russian Federation \\ 2. Lomonosov Moscow State University, Leninskie gory, 1-2, Moscow 119991, Russian Federation
}

Protein adsorption on solid surfaces is widespread in nature and can be utilized in technological applications [1]. In particular, adsorption of blood proteins to a surface may determine the immune response of an organism and, therefore, biocompatibility of a material [2]. Though there is a general understanding that many surfaces may induce structural rearrangements of adsorbed proteins [3], this problem remains poorly studied at a single molecule level for many medically relevant proteins. In this concern, high resolution atomic force microscopy (AFM) investigation of adsorption of blood proteins on graphitic materials, which are very promising for biological and medical applications [4,5], represents an important task in fundamental and applied science. Recently we have visualized quite fast (within $1 \mathrm{sec}$ ) denaturation of some plasma proteins upon their adsorption onto freshly cleaved highly oriented pyrolytic graphite (HOPG) surface [6]. The aim of the current work was AFM investigation of adsorption of fibrinogen on HOPG surface modified with amphiphilic oligoglycine-hydrocarbon graphite modificator (GM), which was shown to preserve the structure of fibrinogen during first seconds of its adsorption [6].

For protein deposition, $0.5 \mu \mathrm{l}$ of $20 \mu \mathrm{g} / \mathrm{ml}$ human fibrinogen (Calbiochem, Germany) solution in $10 \mathrm{mM}$ sodium phosphate buffer ( $\mathrm{pH}$ 7.2) was deposited onto GM-HOPG surface for $10 \mathrm{sec}$. After that, $100 \mu 1$ of deionized water was placed on the sample surface and removed in $0-10$ min by a nitrogen flow. AFM experiments were conducted using the multimode atomic force microscope Ntegra Prima (NTMDT, Russia) equipped by ultra-sharp tips (carbon nanowhiskers grown on the top of commercially available silicon cantilevers [7]) and operated in intermittent contact mode in air at a room temperature with pixel resolution of $1 \mathrm{pixel} / \mathrm{nm}$.

AFM images of fibrinogen molecules adsorbed on GM-HOPG for 10 seconds represent typical trinodular structure, possessing two outer globular regions and a central globular region (fig. 1a) with their corresponding heights of $3.0 \pm 0.3$ and $1.6 \pm 0.4 \mathrm{~nm}$. Moreover, $\alpha \mathrm{C}$ regions (dashed arrows) and subglobular features of outer globular regions ( $\beta$ - and $\gamma$-nodules; solid arrow in (a)) can be also revealed. The fraction of thin $(<1.5 \mathrm{~nm}$ in height) isolated filaments constitutes about $4 \%$. However, for longer periods of fibrinogen adsorption (1-10 $\mathrm{min})$ the increase of the fraction and length of filamentary structures and decrease - of globular ones is observed in corresponding AFM images (fig. 1b-d).

In particular, the fraction of isolated filamentary structures has increased from $\approx 11 \%$ for 1 minute to $\approx 63 \%$ for 10 minutes of fibrinogen adsorption. Most of the fibrinogen molecules after 3-10 min of adsorption do not reveal globular regions but only filamentous structures. Furthermore, there is an evident relationship between the configuration of the observed filaments (fig. 1c,d) and nativelike conformation of a fibrinogen molecule (fig. 1a): a pseudo-symmetry around the central point at the position of a central globule (dotted arrows) and fibril branching at the position of outer globular regions (solid arrows) are observed. The morphology observed in fig. 1b,c may correspond to the 
unfolded (i.e. without tertially structure) chain of fibrinogen molecules anchored with 29 disulfide bonds present in fibrinogen [8].

Due to relatively large time scale (minutes) of unfolding process and partial retaining of unfolded fibrinogen conformation (e.g., of its secondary structure represented by fibrils), the observed GM-HOPG induced unfolding may be considered as mild in comparison with fast and severe fibrinogen denaturation on freshly cleaved HOPG [6]. The obtained results represent the first example of visualization of gradual unfolding of fibrinogen molecule at a single molecule level and may provide better understanding of this process. This system may also be used as a convenient model for real-time AFM investigation of fibrinogen unfolding in solution and for the study of influence of different physicochemical factors on this process [9].

\section{References:}

[1] M Rabe, D Verdes and S Seeger, Adv. Colloid Interface Sci. 162 (2011), p. 87.

[2] KD Jandt, Adv. Eng. Mater. 9 (2007), p. 1035.

[3] K Nakanishi, T Sakiyama and K Imamura, J. Biosci. Bioeng. 91 (2001), p. 233.

[4] K Kostarelos and KS Novoselov, Science 344 (2014), p, 261.

[5] V Pesakova et al, J. Mater. Sci.-Mater. Med. 11 (2000), p. 793.

[6] NA. Barinov et al, Colloids Surf. B Biointerfaces 146 (2016), p. 777.

[7] D Klinov and S Magonov, Appl. Phys. Lett. 84 (2004), p. 2697.

[8] A Zhmurov et al, Structure 24 (2016), p. 1907.

[9] The authors acknowledge funding from the Russian Science Foundation [17-75-30064 to D.V.K.].

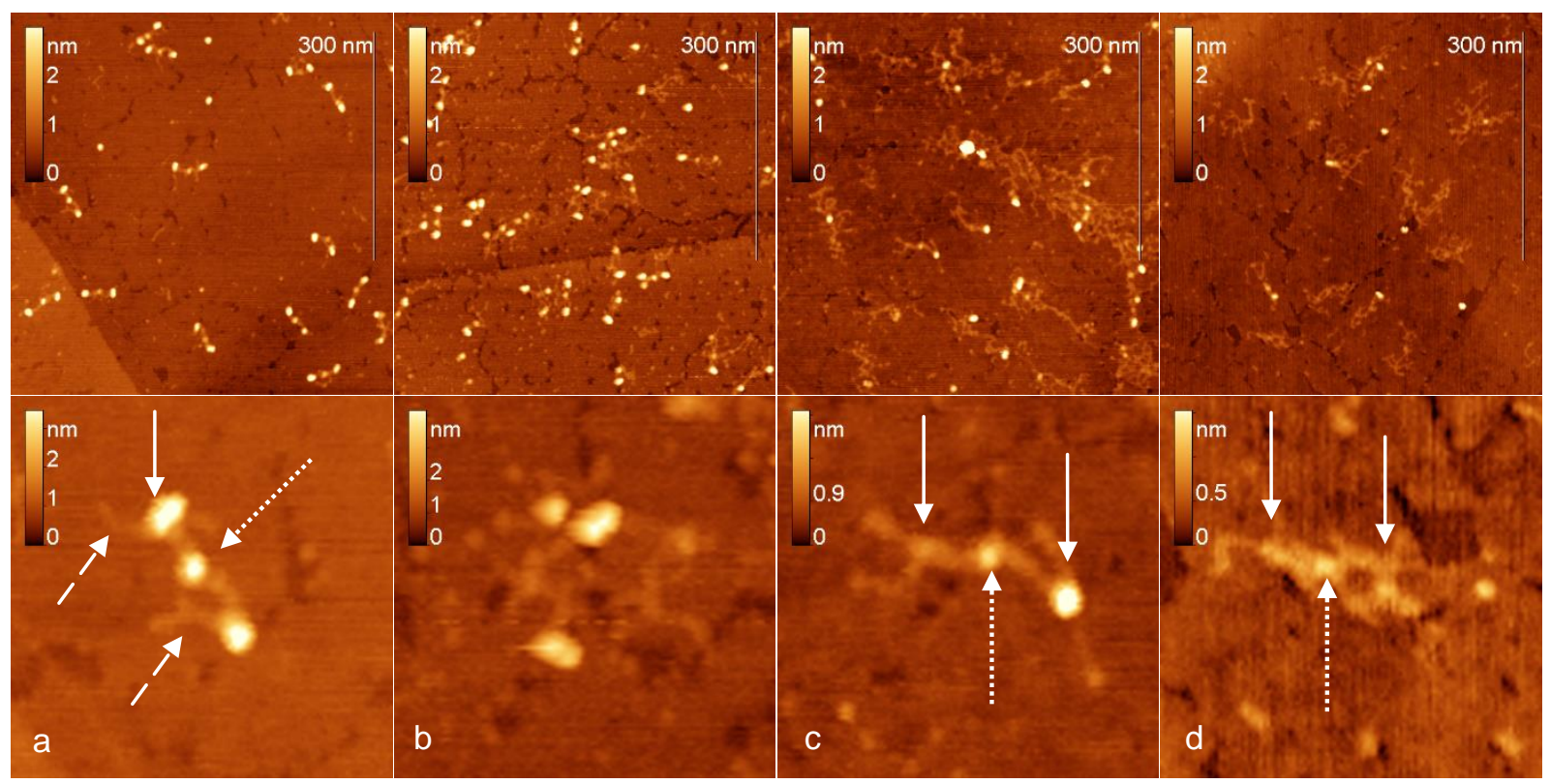

Figure 1. AFM images of fibrinogen molecules adsorbed on GM-HOPG surface for (a) 10 sec, (b) $1 \mathrm{~min}$, (c) $3 \mathrm{~min}$, (d) $10 \mathrm{~min}$. Bottom row represents enlarged regions $\left(100 \times 100 \mathrm{~nm}^{2}\right)$ from the corresponding AFM images on top $\left(500 \times 500 \mathrm{~nm}^{2}\right)$. 